\title{
List of cases of the Court of Justice of the European Union (CJEU)
}

Note: Electronic Reports of Cases (ECLI) numbers are given for cases not published in the printed reports.

C-21/76 Handelskwekerij G. J. Bier BV v Mines de potasse d'Alsace SA, 30 November 1976, [1976] ECR 01735 $232,249,524$

C-352/85 Bond van Adverteerders and others v The Netherlands State, 26 April 1988, [1988] ECR 02085 65

C-189/87 Athanasios Kalfelis v Bankhaus Schröder, Münchmeyer, Hengst and Co. and others, 27 September 1988, [1988] ECR 5565 ....................................232

C-382/87 R. Buet and Educational Business Services (EBS) v Ministère public, 16 May 1989, [1989] ECR 01235

C-220/88 Dumez France and Others v Hessische Landesbank and Others, 11 January 1990, [1990] ECR 1-49. $233,240,524$

C-76/90 Manfred Säger v Dennemeyer \& Co. Ltd., 25 July 1991, [1991] ECR I-4221

C-6/90 and C-9/90 Andrea Francovich and Danila Bonifaci and others v Italian

Republic, 19 November 1991, [1991] ECR I-5357 ....................................348

C-26/91 Jakob Handte \& Co. GmbH v Traitements Mécano-chimiques des Surfaces SA, 17 June 1992, [1992] ECR I-03967.

C-89/91 Shearson Lehmann Hutton v TVB Treuhandgesellschaft fur

Vermogensverwaltung und Beteiligungen, 19 January 1993, [1993] ECR I-139.....

C-275/92 HM Customs and Excise v Gerhart Schindler and Jörg Schindler, 24

March 1994, [1994] ECR I-01039 308

C-359/92 Germany v Council, 9 August 1994, [1994] ECR I-3683. .354

C-68/93 Fiona Shevill, Ixora Trading Inc., Chequepoint SARL and Chequepoint International Ltd v Presse Alliance SA, 7 March 1995, [1995]

ECR I-415 $199,233,246,524$

C-364/93 Marinari v Lloyd's Bank, 19 September 1995, [1995] ECR 1-6511 ....233, 240,524

C-269/95 Francesco Benincasa v Dentalkit Srl, 3 July 1997, [1997] ECR I-3767 262 C-337/95 Parfums Christian Dior SA and Parfums Christian Dior BV v Evora BV, 4

November 1997, [1997] ECR I-6013 159

C-251/95 Sabel BV v Puma AG, Rudolf Dassler Sport, 11 November 1997, [1997]

ECR I-6191 162

C-39/97 Canon Kabushiki Kaisha v Metro-Goldwyn-Mayer Inc., formerly Pathe

Communications Corporation, 29 September 1998, [1998] ECR I-5507 ....156, 
C-63/97 Bayerische Motorenwerke AG (BMW) and BMW Nederland BV v Ronald Karel Deenik, 23 February 1999, [1999] ECR I-905 ..................................153

C-99/96 Hans-Hermann Mietz v Intership Yachting Sneek BV, 27 April 1999,

[1999] ECR I-2277

C-124/97 Markku Juhani Läärä, Cotswold Microsystems Ltd and Oy Transatlantic

Software Ltd v Kihlakunnansyyttäjä (Jyväskylä) and Suomen valtio (Finnish

State), 21 September 1999, [1999] ECR I-06067....

C-67/98 Questore di Verona v Diego Zenatti, 21 October 1999, [1999] ECR

I-07289.

C-453/99 Courage Ltd v Bernard Crehan and Bernard Crehan v Courage Ltd and

Others, 20 September 2001, [2001] ECR I-6297.

C-481/99 Georg Heininger and Helga Heininger v Bayerische Hypo- und

Vereinsbank AG, 13 December 2001, [2001] ECR I-09945

C-167/00 Verein für Konsumenteninformation v Karl Heinz Henkel, 1 October 2002, [2002] ECR I-08111

C-206/01 Arsenal Football Club plc v Matthew Reed, 12 November 2002, [2002]

ECR I-10273. $.152,389$

C-245/00 Stichting ter Exploitatie van Naburige Rechten (SENA) v Nederlandse

Omroep Stichting (NOS), 6 February 2003, [2003] ECR I-01251 ..............134

C-291/00 LTJ Diffusion SA v Sadas Vertbaudet SA, 20 March 2003, [2003] ECR I-2799.

C-112/00 Eugen Schmidberger, Internationale Transporte und Planzüge v Republik

Österreich, 12 June 2003, [2003] I-05659. .528

C-408/01 Adidas-Salomon and Adidas Benelux v Fitness- world, 23 October 2003,

[2003] ECR I-2537

C-243/01 Criminal proceedings against Piergiorgio Gambelli and Others, 6

November 2003, [2003] ECR I-13031

C-322/01 Deutscher Apothekerverband eV v 0800 Doc Morris NV and Jacques

Waterval, 11 December 2003, [2003] ECR I-14887....

C-18/02 Danmarks Rederiforening, acting on behalf of DFDS Torline A/S v LO

Landsorganisationen i Sverige, acting on behalf of SEKO Sjöfolk Facket för

Service och Kommunikation, 5 February 2004, [2004] ECR 1-01417.240, 524

C-168/02 Rudolf Kronhofer v Marianne Maier and Others, 5 February 2004, [2004]

ECR 1-6009 $.240,524$

C-245/02 Anheuser-Busch Inc. v Budejovický Budvar, národní podnik, 16

November 2004, [2004] ECR I-10989 .......................................................158

C-464/01 Johann Gruber v Bay Wa AG, 20 January 2005, [2005] ECR I-439 ......262

C-27/02 Petra Engler v Janus Versand GmbH, 20 January 2005, [2005]

I-00481.....

C-212/03 Commission v France, 26 May 2005, [2005] ECR I-4213 .......................307

C-89/04 Mediakabel BV v Commissariaat voor de Media, 2 June 2005, [2005] ECR I-04891 .

C-361/04 P Claude Ruiz-Picasso and Others v Office for Harmonisation in the

Internal Market (Trade Marks and Designs), 12 January 2006, [2006] ECR

I-643.

C-4/03 Gesellschaft für Antriebstechnik mbH \& Co. KG v Lamellen und

Kupplungsbau Beteiligungs KG(GAT), 13 July 2006, [2006] ECR 6509...215, 
C-295/04, C-296/04, C-297/04 and C-298/04 Vincenzo Manfredi v Lloyd Adriatico Assicurazioni SpA, Antonio Cannito v Fondiaria Sai SpA, Nicolò Tricarico and Pasqualina Murgolo v Assitalia SpA, 13 July 2006, [2006] ECR I-6619.

C-48/05 Adam Opel AG v Autec AG, 25 January 2007, [2007] ECR I-1017........153, 155,390

C-17/06 Céline SARL v Céline SA, 11 September 2007, [2007] ECR I-7041 ......155

T-201/04 Microsoft v Commission, 17 September 2007, [2007] II-03601 ............108

C-275/06 Productores de Música de España (Promusicae) v Telefónica de España

SAU, 29 January 2008, [2008] ECR I-271 $.98,118,324$

C-404/06 Quelle AG v Bundesverband der Verbraucherzentralen und

Verbraucherverbände, 17 April 2008, [2008] ECR I-2685 .

C-533/06 O2 Holdings Ltd v Hutchison 3G UK Ltd, 12 June 2008, [2008] E.C.R. I-4231

C-298/07 Bundesverband der Verbraucherzentralen und Ver- braucherverbände -

Verbraucherzentrale Bundesverband $\mathrm{eV}$ v. deutsche Internet versicherung AG,

16 October 2008, [2008] ECR I-07841

C-252/07 Intel Corp Inc v CPM United Kingdom Ltd, 27 November 2008, [2008]

ECR I-8823.

C-557/07 LSG-Gesellschaft zur Wahrnehmung von Leistungsschutzrechten, 19

February 2009 [2009] ECR I-01227 .

C-62/08 UDV North America v Brandtraders NV, 19 February 2009, [2009] ECR I-1279. .396

C-59/08 Copad SA v Christian Dior couture SA, Vincent Gladel and Société industrielle lingerie (SIL), 23 April 2009, [2009] ECR I-3421..

C-261/07 and C-299/07 Mediaprint; Zentrale zur Bekämpfung unlauteren

Wettbewerbs eV; Galatea BVBA v Sanoma Magazines Belgium NV, 23 April

2009, [2009] ECR I-02949

C-204/08 Rehder v Air Baltic, 9 July 2009, [2009] ECR I-6073 ..........................263

C-5/08 Infopaq International A/S v Danske Daglades Forening (Infopaq I), 16 July 2009, [2009] ECR I-6569. $86,97,117$

C-189/08 Zuid-Chemie BV v Philippo's Mineralenfabriek NV/SA, 16 July 2009, [2009] ECR I-6917 232,236

C-489/07 Pia Messner v Firma Stefan Krüger, 3 September 2009, [2009] I-07315

C-424/07 Commission v. Germany, 3 December 2009, [2009] ECR I-11431 .......344

C-304/08 Zentrale zur Bekämpfung unlauteren Wettbewerbs eV v Plus

Warenhandelsgesellschaft mbH., 14 January 2010, [2010] ECR I-217 ........347

C-555/07 Seda Kücükdeveci v Swedex GmbH \& Co. KG., 19 January 2010, [2010] $\mathrm{I}-00365$.

C-317/08, C-318/08, C-319/08 and C-320/08 Rosalba Alassini v Telecom Italia SpA, Filomena Califano v Wind SpA, Lucia Anna Giorgia Iacono v Telecom Italia SpA and Multiservice Srl v Telecom Italia SpA, 18 March 2010, [2010]

ECR I-2213. 354

C-236/08 and 238/08 Google France SARL and Google Inc. v Louis Vuitton Malletier SA and Others, 23 March 2010, [2010] ECR I-2417. $.9,144$ 149-52, 155-6, 159, 163-5, 225, 238, 389-96, 535 
C-278/08 Die BergSpechte Outdoor Reisen und Alpinschule Edi Koblmüller GmbH v Günther Guni and trekking.at Reisen GmbH, 25 March 2010, [2010] ECR I-2517 $144,154,156,159$

C-558/08 Portakabin Ltd and Portakabin BV v Primakabin BV, 8 July 2010, [2010] ECR I-6963. $144,152,154,156,158,165-6,390,394$

C-467/08 Padawan SL v Sociedad General de Autores y Editores de España (SGAE), 21 October 2010, [2010] ECR I-10055 $.131,133$

C-540/08 Mediaprint Zeitungs- und Zeitschriftenverlag GmbH \& Co. KG v 'Österreich'-Zeitungsverlag GmbH., 9 November 2010, [2010] ECR I-10909 347,425

C-108/09 Ker-Optika bt v ÀNTSZ Dél-dunántúli Regionális Intézete, 2 December 2010, [2010] ECR I-12213 308

C-144/09 Hotel Alpenhof GESMBH vs. Oliver Heller and C-585/08 Peter Pammer vs. Reederie Karl Schlüter GMBH \& CO KG, 7 December 2010, [2010] ECR I-12527 $202,212-13,223,235,249,263$

C-393/09 Bezpečnostní softwarová asociace - Svaz softwarové ochrany v Ministry of Culture of the Czech Republic, 22 December 2010, [2010] ECR I-13971 97

C-235/09 DHL Express France SAS v Chronopost SA, 12 April 2011, [2011] ECR I-02801 . .202

C-122/10 Konsumentombudsmannen v Ving Sverige AB, 12 May 2011, [2011] ECR I-03903... $.346-7$

C-65/09 and C-87/09 Gebr. Weber GmbH v Jürgen Wittmer and Ingrid Putz v Medianess Electronics GmbH, 16 June 2011, [2011] ECR I-5257 ...............351

C-462/09 Stichting deThuiskopie v. Opus Supplies Deutschland GmbH, M. van der lee, H. van der Lee, 16 June 2011, [2011] ECR I-05331 .... 131

C-324/09 L'Oréal SA, Lancôme parfums et beauté \& Cie SNC, Laboratoire Garnier \& Cie, L'Oréal (UK) Ltd v eBay International AG et al, 12 July 2011, [2011] ECR I-06011 ..............................9, 145, 149-53, 163, 235, 318-20, 396, 535

C-323/09 Interflora e.a. v. Marks \& Spencers, 22 September 2011, [2011] ECR I-08625 $144,152,155-7,160-61,163-5,390$

C-403/08 Football Association Premier League Ltd and Others v QC Leisure and Others and C-429/08 Karen Murphy v Media Protection Services Ltd., 4 October 2011, [2011] ECR I-09083. $86,117,119,122$

C-439/09 Pierre Fabre Dermo-Cosmétique SAS v Président de l'Autorité de la concurrence and Ministre de l'Économie, de l'Industrie et de l'Emploi, 13 October 2011, [2011] ECR I-09419 .308

C-509/09 and C-161/10 eDate Advertising GmbH v X, Olivier Martinez, Robert Martinez v MGN Limited, 25 October 2011, [2011] ECR I-10269 .....200-201, 226, 231, 234-6, 239, 243-5, 248, 250, 525

C-70/10 Scarlet Extended SA v Société belge des auteurs, compositeurs et éditeurs SCRL (SABAM) 24 November 2011, [2011] ECR I-11959. $9,98,323-6$, $327-8,536$

C-406/10 SAS Institute Inc. v World Programming Ltd, 29 November 2011, ECLI:EU:C:2012:259. 92,97

C-302/10 Infopaq International A/S v Danske Dagblades Forening (Infopaq II),

17 January 2012, ECLI:EU:C:2012:16 $86,117,122$

C-277/10 Martin Luksan v Petrus van der Let, 9 February 2012, ECLI:EU:C:2012:65 
C-360/10 Belgische Vereniging van Auteurs, Componisten en Uitgevers CVBA

(SABAM) v Netlog NV, 16 February 2012, ECLI:EU:C:2012:85 .......325, 536

C-604/10 Football Dataco Ltd. V Yahoo! Uk Ltd, 1 March 2012,

ECLI:EU:C:2012:115

C-461/10 Bonnier Audio AB v. Perfect Communication Sweden AB, 19 April 2012,

ECLI:EU:C:2012:219. 49,98

C-523/10 Wintersteiger AG v Products 4U Sondermaschinenbau GmbH, 19 April 2012, ECLI:EU:C:2012:220 201, 231, 234-45, 248, 250-53

C-618/10 Banco Español de Crédito SA v Joaquín Calderón Camino, 14 June 2012, ECLI:EU:C:2012:349 485

C-128/11 UsedSoft GmbH v Oracle International Corp, 3 July 2012, ECLI:EU:C:2012:407

C-616/10 Solvay SA v Honeywell Fluorine Products Europe BV and Others, 12 July 2012, ECLI:EU:C:2012:445

C-173/11 Football Dataco Ltd and Others v Sportradar GmbH and Sportradar AG, 18 October 2012, ECLI:EU:C:2012:3823........196-7, 224-5, 231, 234, 241-3,

C-145/10 Eva-Maria Painer v Standard VerlagsGmbH and Others, 7 March 2013, ECLI:EU:C:2013:138. 97,119

C-92/11 RWE Vertrieb AG v Verbraucherzentrale Nordrhein-Westfalen eV., 21

March 2013, ECLI:EU:C:2013:180 485

C-274/11 and C-295/11 Kingdom of Spain and Italian Republic v Council of the European Union, 16 April 2013, ECLI:EU:C:2013:240 ....

C-228/11 Melzer v MF Global UK Ltd, 16 May 2013, ECLI:EU:C:2013:305 .....247

C-279/13 C More Entertainment AB v Linus Sandberg, lodged on 22 May 2013

C-488/11 Dirk Frederik Asbeek Brusse and Katarina de Man Garabito v Jahani BV., 30 May 2013, ECLI:EU:C:2013:341

C-348/13 BestWater International GmbH v Michael Mebes, Stefan Potsch, lodged on 25 June 2013 124,125

C-457/11, C-458/11, C-459/11 and C-460/11 Verwertungsgesellschaft Wort (VG Wort) v Kyocera and Others; Canon Deutschland GmbH and Fujitsu Technology Solutions GmbH and Hewlett-Packard GmbH v Verwertungsgesellschaft Wort (VG Wort), 27 June 2013, ECLI:EU:C:2013:426. 112,131

C-521/11 Amazon.com International Sales Inc. and Others v Austro-Mechana Gesellschaft zur Wahrnehmung mechanisch-musikalischer Urheberrechte Gesellschaft mbH, 11 July 2013, ECLI:EU:C:2013:515. 131,133

C-170/12, Peter Pinckney v KDG Mediatech AG, 3 October 2013, ECLI:EU:C:2013:635 $201-2,231,243-53$

C-508/12 Walter Vapenik v Josef Thurner, 5 December 2013, ECLI:EU:C:2013:790

C-466/12 Nils Svensson, Sten Sjögren, Madelaine Sahlman, Pia Gadd v. Retreiver Sverige AB, 13 February 2014, ECLI:EU:C:2014:76 $124,125,192$

C-314/12 UPC Telekabel Wien GmbH v Constantin Film Verleih GmbH and Wega Filmproducktionsgesellschaft mbH., 27 March 2014, ECLI:EU:C:2014:192 $192,313,537$ 
C-293/12 and C-594/12 Digital Rights Ireland Ltd v Minister for Communications and Kärntner Landesregierung, Michael Seitlinger, Christof Tschohl and others, 8 April 2014, ECLI:EU:C:2014:238. $.303,539$

C-435/12 ACI Adam BV and Others v Stichting de Thuiskopie and Stichting Onderhandelingen Thuiskopie vergoeding, 10 April 2014, ECLI:EU:C:2014:254

C-131/12 Google Spain SL and Google Inc. v Agencia Española de Protección de Datos (AEPD) and Mario Costeja González, 13 May 2014, ECLI:EU:C:2014:616.... $.538,556-7$

C-360/13 Public Relations Consultants Association Ltd v The Newspaper Licensing Agency Ltd and others, 5 June 2014, ECLI:EU:C:2014:910 125 